\title{
Role of Capsule in Pulmonary Hypertension Induced by Group B Streptococcus
}

\author{
JOSEPH B. PHILIPS, III, JIA-XIAN LI, BARRY M. GRAY, DAVID G. PRITCHARD, AND \\ JAMES R. OLIVER
}

Perinatal Physiology Research Laboratory, Division of Neonatology, Department of Pediatrics [J.B.P., J.-X.L.,

J.R.O.], Division of Pediatric Infectious Diseases, Department of Pediatrics [B.M.G., D.G.P.], and Department of Microbiology [B.M.G., D.G.P.], University of Alabama at Birmingham, Alabama 35233-7335

\begin{abstract}
The type-specific polysaccharide capsule of group B streptococcus (GBS) is thought to be an important factor in the pathogenesis of disease. We used an acutely instrumented piglet model to assess the hemodynamic effects of rapid infusions of two heat-killed GBS type Ib strains isolated from the spinal fluid of an infant with lateonset meningitis and from the vaginal culture of his mother. These strains expressed different amounts of capsule, as determined by buoyant density centrifugation and electron micrographs, and they produced different hemodynamic effects in the piglets. The mother's strain, which had a smaller capsule, caused significantly higher increases in pulmonary artery pressure and vascular resistance than did the infant's strain, which had a larger capsule. Transposon mutants were then made from the infant's isolate to further study the role of capsule in pulmonary hypertension. Two mutants lacking detectable capsular type-specific polysaccharide were compared with the original isolate and with an isogenic mutant containing transposons but having a large capsule. The nonencapsulated mutants caused significantly higher changes in pulmonary artery pressure and resistance than did the encapsulated strains. Pulmonary hypertension may play a role in the pathophysiology of GBS sepsis, but the presence of a large capsule may partially cloak the hemodynamically active component(s) of the bacteria. The lower initial host response to heavily encapsulated GBS may play a role in pathogenesis by helping the organisms avoid host defense mechanisms. (Pediatr Res 31: 386-390, 1992)
\end{abstract}

\section{Abbreviations}

CDM, chemically defined medium EM, electron micrograph GBS, group B streptococcus $\mathrm{PH}$, pulmonary hypertension Ppa, pulmonary artery pressure

Psa, systemic artery pressure Pla, left atrium pressure Pcv, central venous pressure

Qpa, mean pulmonary artery flow Rpv, pulmonary vascular resistance Rsv, systemic vascular resistance cfu, colony forming units

Group B streptococci are the most common cause of serious neonatal bacterial infections in the United States and have been reported to cause neonatal disease in many countries throughout the world (1-3). Pulmonary hypertension may be observed in

Received June 14, 1991; accepted November 27, 1991.

Correspondence and reprint requests: Joseph B. Philips, III, M.D., University of Alabama at Birmingham, 525 New Hillman Building, UAB Station, Birmingham AL 35233-7335. human neonates with GBS sepsis $(2,4)$ and can be induced in animal models by infusion of GBS $(5,6)$. Despite many improvements in therapy and supportive care, the mortality from GBS sepsis remains unacceptably high (7). Many reports indicate that GBS colonization of the maternal birth canal is the most important source for neonatal sepsis $(8-10)$. Studies of mother-infant pairs of vertically transmitted GBS have demonstrated significant differences between some of the pairs in the expression of typespecific capsular polysaccharide (11). The presence of a large capsule is thought to be an important factor in pathogenesis and has been shown to be associated with increased virulence in a mouse model (12). Most virulence studies in animals have used death as an end point, rather than physiologic variables. Studies comparing the hemodynamic effects of paired isolates from infected infants and their mothers have not been reported. We used a piglet model to test the hypothesis that the hemodynamic effects of a pair of GBS type Ib isolates from an infected infant and its colonized mother that vary in the amount of capsular polysaccharide would be different. Isogenic transposon mutants of the infant's strain were then used to further study the role of capsule in the hemodynamic changes induced by these otherwise genetically similar GBS.

\section{MATERIALS AND METHODS}

Animal preparation. Fifteen female piglets were anesthetized with halothane $(5 \%$ for induction, $1-1.5 \%$ for surgery) and mechanically ventilated through an endotracheal tube using standard anesthesia equipment with $100 \%$ oxygen. Animals were placed on a heating pad to maintain body temperature at $37 \pm$ $1^{\circ} \mathrm{C}$. A left thoracotomy was performed, and the pericardium was opened. A $10-\mathrm{mm}$ precalibrated ultrasonic flow transducer (Transonic Systems, Ithaca, NY) was placed around the main pulmonary artery. A 5 Fr polyurethane catheter (Sherwood Medical, St. Louis, MO; courtesy of Mr. Ray Bodicky) was then inserted into the pulmonary artery between the pulmonary valve and the flow probe. Another 5 Fr catheter was placed in the left atrium. Finally, aortic and inferior vena cava catheters were placed through a left groin incision. All catheters were flushed with 10 units/mL heparin in normal saline. After surgery, which typically lasted for $1 \mathrm{~h}$, pentobarbital was injected i.v. $(10 \mathrm{mg} / \mathrm{kg}$ every hour) instead of halothane to maintain sedation during the experiment period. A stabilization period of at least $1 \mathrm{~h}$ was allowed between completion of surgery and the start of experiments. Inspired oxygen concentration remained at $100 \%$ throughout experimentation. Arterial blood gases were measured and the ventilator was adjusted and/or sodium bicarbonate was given to achieve an arterial $\mathrm{pH}$ of 7.30-7.40 with an arterial $\mathrm{CO}_{2}$ tension between 4.0 and $5.4 \mathrm{kPa}$ (30 and 40 torr). Ppa, Psa, Pla, and $\dot{Q}$ pa were continually monitored using precalibrated transducers and a physiologic recorder (Gould-Brush 2400, Oxnard, 
CA). All monitored variables were quite stable during the preexperiment period.

Organism preparation. A pair of GBS type Ib strains were isolated from the cerebrospinal fluid of an infant with late-onset meningitis and from the vagina of his healthy mother at the time of the infant's admission. Three isogenic mutants were made from the infant's strain by inserting the modified transposon $\operatorname{Tn} 916 \Delta \mathrm{E}$ (13). Southern hybridization of the chromosomal DNA of the mutants was done using the plasmid pVA 891 as a probe for the erythromycin resistance gene of the transposon (14). Multiple transposon insertions are typical when $\operatorname{Tn} 916 \Delta \mathrm{E}$ is introduced into the acceptor strain by the process of conjugation (15). Mutant 49-2 contained two transposons but had a normal large capsule and was used as a control for the two nonencapsulated mutants, 36-1 and 37-5, which contained two and four transposons, respectively. Neither of the latter two strains produced any detectable type-specific polysaccharide by immunodiffusion tests with mutanolysin-digested bacteria and type-specific rabbit or mouse MAb.

Organisms prepared for infusion into piglets were grown to late log phase (4-5 h) in CDM. The cultures were heat-killed at $80^{\circ} \mathrm{C}$ for $30 \mathrm{~min}$, washed twice in PBS, and resuspended in PBS to an absorbance of 0.6 at $590 \mathrm{~nm}$. This corresponds to approximately $1 \times 10^{9} \mathrm{cfu} / \mathrm{mL}$ as determined by colony counts of GBS processed similarly but without heat killing. Microscopic examination revealed that about half of the organisms were present as diplococci, with the remainder as short chains of three to eight cocci. Aliquots of these preparations were stored at $-70^{\circ} \mathrm{C}$ until thawed and were mixed thoroughly just before use. Each set of experiments was done with the same set of frozen GBS. The microscopic appearance and density of GBS thus prepared and rethawed was similar to that observed before heat killing.

Organism characterization. The amount of capsular material was estimated by buoyant density centrifugation essentially as described by Håkansson and Holm (16). GBS were grown for 24 $h$ in CDM (17), harvested by centrifugation, washed once with PBS $(0.05 \mathrm{M}$ phosphate, $0.15 \mathrm{M} \mathrm{NaCl}$, pH 7.4) and twice with distilled water, applied to $20 \mathrm{~mL}$ Percoll (Pharmacia Fine Chemicals, Piscataway, NJ) gradients, and centrifuged at $12000 \times g$ for $20 \mathrm{~min}$. The density was measured by the final position of the bacterial band in the gradient, relative to that of colored beads (Pharmacia) of densities measured independently in distilled water by a pyknometer method.

Bacteria were prepared for electron microscopy after overnight culture in CDM, then were centrifuged and washed twice in EM buffer $(0.05 \mathrm{M}$ Tris, $0.15 \mathrm{M} \mathrm{NaCl}, 1 \% \mathrm{BSA}, \mathrm{pH}$ 7.4). The organisms were resuspended for $30 \mathrm{~min}$ at room temperature in mouse ascites fluid containing MAb SmB19 against the complete type Ib capsular polysaccharide. After washing in EM buffer, gold-conjugated goat anti-mouse Ig (Zymed, South San Francisco, CA) was added and incubated for $30 \mathrm{~min}$, and washed as before. The bacteria were then fixed with $4 \%$ glutaraldehyde, dehydrated through graded alcohol and 2,2-dimethoxypropane, embedded in Spurr ultra-light medium (Ladd Research Industries, Burlington, VT), sectioned, and viewed with a transmission electron microscope (Philips 301, Eindhoven, The Netherlands).

Experiment 1. Seven piglets $10-15 \mathrm{~d}$ of age weighing $4.1 \pm 0.3$ $\mathrm{kg}$ (mean $\pm \mathrm{SD})$ were rapidly $(<3 \mathrm{~s})$ injected with boluses given in random order of each of the mother's and infant's GBS strains through the inferior vena cava catheter at a dose of $1 \times 10^{8} \mathrm{cfu} /$ $\mathrm{kg}$. Only one dose of each organism was given. The next injection was not made until Ppa had returned to baseline for 10 or more $\min$.

Experiment 2. Another eight piglets aged $10-21 \mathrm{~d}$ weighing $4.5 \pm 0.8 \mathrm{~kg}$ were injected i.v. with boluses $\left(1 \times 10^{8} \mathrm{cfu} / \mathrm{kg}\right)$ of the infant's strain and the three isogenic mutants, randomized by Latin square design. The remainder of the experimental program was the same as in experiment 1.

Data management. All physiologic values were simultaneously read before each injection of GBS and at the peak response of
Ppa after injection of GBS. Mean pressures $(\overline{\mathrm{P}})$ were calculated in the standard fashion. Rpv was calculated as $0.6(\overline{\mathrm{P} p a}-\overline{\mathrm{P}} \mathrm{la}) /$ Qpa, and Rsv was calculated as $0.6(\overline{\mathrm{P}} \mathrm{pa}-\overline{\mathrm{P}} \mathrm{cv}) / \mathrm{Qpa}$ in experiment 1 and as $0.6(\overline{\mathrm{P}} \mathrm{sa} / \mathrm{Qpa})$ in experiment 2 . The paired $t$ test was used for comparison of responses between mother's and infant's strains. Three-way analysis of variance was used to evaluate GBS-related hemodynamic responses in experiment 2. Duncan's multiple-range test was then used to compare each capsule-deficient mutant strain against the original infant's strain and mutant $49-2$. A $p$ value $\leq 0.05$ was considered statistically significant.

\section{RESULTS}

Characterization of bacterial strains. Buoyant density studies showed that the infant's strain had a density of $1.044 \mathrm{~g} / \mathrm{mL}$ as a result of a large capsule, whereas the mother's strain had a density of $1.081 \mathrm{~g} / \mathrm{mL}$, indicating a smaller capsule. The two nonencapsulated strains, 36-1 and 37-5, had buoyant densities of 1.088 and $1.082 \mathrm{~g} / \mathrm{mL}$, respectively. Mutant 49-2, which contained transposons but expressed a large capsule, had a buoyant density of $1.045 \mathrm{~g} / \mathrm{mL}$.

EM of three of the GBS strains are shown in Figure 1. The infant's strain (left) had a large capsule stabilized by antibody surrounding the bacterial body. The mother's strain (center) had a much smaller capsule, with polysaccharide identified by the immunogold beads. The nonencapsulated mutant 37-5 (right) had no visible capsule and no type-specific polysaccharide detected by immunogold labeling.

General response to bacterial injections. All GBS injections caused transient effects on some of the monitored variables, especially $\overline{\mathrm{P}} \mathrm{pa}$ and Q pa (Fig. 2). The rise in $\overline{\mathrm{P}}$ pa typically began about $30 \mathrm{~s}$ after injection and peaked at 50-100 s. Values then

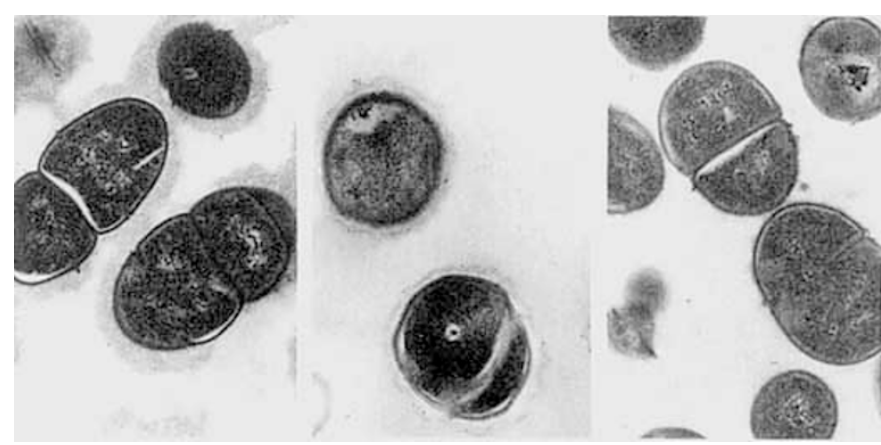

Fig. 1. Immunoelectron micrographs showing the large-capsule infant's GBS strain (left), the smaller capsule mother's strain (middle), and nonencapsulated mutant 37-5 (right). Small $(5 \mathrm{~nm}$ ) immunogold beads are bound to capsules of the infant's and mother's strains, but none are seen in the nonencapsulated mutant 37-5.

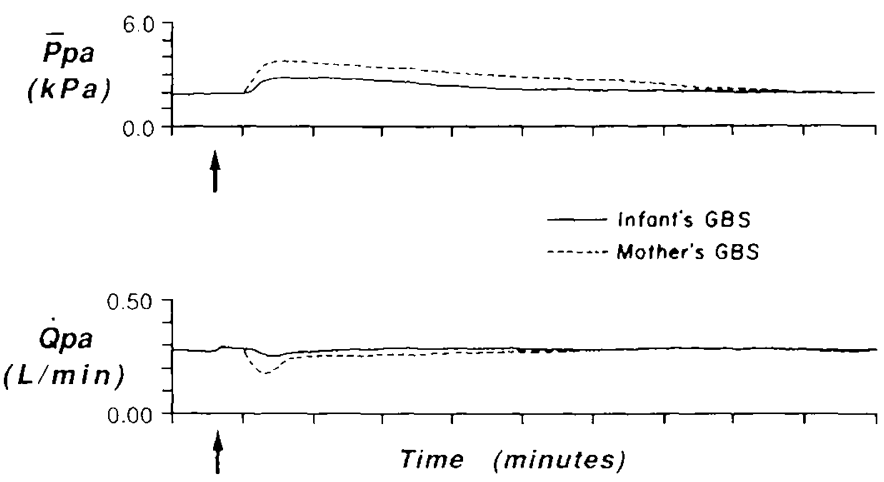

Fig. 2. Actual tracings of the effects of the mother's (-) and infant's (--) GBS strains on Ppa and Qpa. The arrow denotes the time of GBS injection. 
gradually returned to preinfusion baseline values within 5 to 10 $\min$.

Responses to mother's and infant's GBS. Preinjection baseline hemodynamic and blood gas values (not shown) were stable with time; arterial $\mathrm{O}_{2}$ tension remained $>40 \mathrm{kPa}$ (300 torr) throughout experimentation. The results from a typical study in experiment 1 are shown in Figure 2, and all results are presented in Table 1. Both strains caused significant increases in Ppa; however, the increase in $\bar{P}$ pa induced by the mother's strain, which has a small capsule, was significantly higher $(p<0.01)$ than that induced by the infant's larger capsule strain. No significant changes were seen in $\bar{P}$ sa. The infant's strain slightly but significantly decreased $\overline{\mathrm{P}} \mathrm{la}$, whereas $\overline{\mathrm{P}} \mathrm{cv}$ was significantly increased by both strains. Both strains also caused reductions in Qpa and increases in Rpv and Rsv. However, the increase in Rpv induced by the small-capsule mother's strain was significantly greater than that seen with infant's strain.

Responses to infant's strain and mutants. Preinfusion hemodynamic variables and arterial blood gas values were stable throughout each experiment. There was no significant order effect on the responses. Each GBS strain caused a significant rise in Ppa (Table 2). The increases in Ppa induced by mutants 36-1 and 37-5, which lack capsule, were similar to one another and were significantly higher than those induced by the infant's original strain or by the encapsulated mutant $49-2$. $\overline{\text { Psa }}$ was not significantly affected by any of the strains. Mutant 36-1 caused a small but significant decrease in $\bar{P}$ la. Only the nonencapsulated strains caused significant decreases in Qpa, whereas all strains increased Rpv and Rsv. The increases in Rpv effected by the capsule-deficient mutants were more than 2-fold higher than those caused by the large-capsule organisms. Although not compared directly, the nonencapsulated mutants produced about the same hemodynamic changes as did the smaller capsule mother's strain in experiment 1 .

\section{DISCUSSION}

Many studies have now clearly established that the early $\mathrm{PH}$ response induced by GBS injection in animals is mediated via release of thromboxane $A_{2}$. The GBS agent(s) responsible for triggering this thromboxane $\mathrm{A}_{2}$ release has not been definitively identified. Results of this and previous studies in our laboratory support the hypothesis that the hemodynamically active component(s) is expressed on the surface of the GBS organism. The GBS used in these studies were washed twice and resuspended in PBS, thereby eliminating most media components. We previously found that supernates of centrifuged aliquots of GBS suspensions had no hemodynamic activity, whereas the resuspended organisms retained full potency $(18)$.

The GBS strains used in the present studies differ in the quantity of capsular material expressed on the cell surface. The mother-infant pair of GBS strains used in experiment 1 was selected because the original isolates showed a marked difference in capsule size and because this difference in capsule production between the two strains has remained stable over time (Gray BM, Pritchard DG, unpublished observations). Mutant 49-2, which contains transposons but has a large capsule, served as a control strain and caused hemodynamic responses similar to the infant's original strain. Both mutant 36-1 and mutant 37-5 lack detectable capsule and caused significantly greater hemodynamic changes than did the heavily encapsulated strains. These results suggest that the hemodynamically active component(s) of GBS in this model is not capsular type-specific polysaccharide. In fact, the more capsule present, the lower the response. In effect, capsular material appears to partially cloak the hemodynamically active component(s) of GBS, thereby attenuating the acute hemodynamic responses induced by rapid infusion of heat-killed GBS. It is possible, however, that the increased $\mathrm{PH}$ induced by the two mutant organisms was not due to lack of capsular type-specific polysaccharide. Instead, transposon insertion could have caused increased expression of an additional "hemodynamic factor." We think that this is unlikely for the following reasons. First, use of two different mutants, both of which lack type-specific polysaccharide, reduces the likelihood that the additional transposon insertions produced similar mutations in expression of a putative "hemodynamic factor." Second, this and other studies in our laboratory using transposon mutants with large capsules have shown hemodynamic effects similar to nonmutated large capsule organisms (19). Thus, insertion of transposons does not, per se, increase the $\mathrm{PH}$ response caused by injection of the resulting

Table 1. Hemodynamic responses to bolus injections of mother's and infant's $G B S^{*}$

\begin{tabular}{|c|c|c|}
\hline & Infant's strain & Mother's strain \\
\hline \multicolumn{3}{|c|}{$\overline{\mathrm{P} p a}[\mathrm{kPa}(\mathrm{mm} \mathrm{Hg})]$} \\
\hline Baseline & $2.29 \pm 0.29(17 \pm 2)$ & $2.27 \pm 0.28(17 \pm 2)$ \\
\hline Maximum & $3.26 \pm 0.69(24 \pm 5) \dagger$ & $3.90 \pm 0.63(29 \pm 5) \dagger \dagger$ \\
\hline \multicolumn{3}{|c|}{$\overline{\mathrm{P}}_{\mathrm{sa}}[\mathrm{kPa}(\mathrm{mm} \mathrm{Hg})]$} \\
\hline Baseline & $8.98 \pm 1.94(67 \pm 15)$ & $8.84 \pm 2.08(66 \pm 16)$ \\
\hline Maximum & $9.02 \pm 2.08(68 \pm 16)$ & $8.78 \pm 2.34(66 \pm 18)$ \\
\hline \multicolumn{3}{|c|}{$\overline{\mathrm{P}} \mathrm{la}[\mathrm{kPa}(\mathrm{mm} \mathrm{Hg})]$} \\
\hline Baseline & $1.09 \pm 0.20(8.1 \pm 1.5)$ & $1.09 \pm 0.21(8.2 \pm 1.5)$ \\
\hline Maximum & $1.04 \pm 0.21(7.8 \pm 1.6) \S$ & $1.04 \pm 0.23(7.8 \pm 1.8)$ \\
\hline \multicolumn{3}{|c|}{$\overline{\mathrm{P}} \mathrm{cv}[\mathrm{kPa}(\mathrm{mm} \mathrm{Hg})]$} \\
\hline Baseline & $0.74 \pm 0.15(5.6 \pm 1.1)$ & $0.75 \pm 0.14(5.6 \pm 1.0)$ \\
\hline Maximum & $0.80 \pm 0.17(6.0 \pm 1.3) \S$ & $0.81 \pm 0.15(6.0 \pm 1.1) \S$ \\
\hline \multicolumn{3}{|l|}{ Qpa $\left(\mathrm{L} \cdot \min ^{-1}\right)$} \\
\hline Baseline & $0.35 \pm 0.06$ & $0.35 \pm 0.07$ \\
\hline Maximum & $0.32 \pm 0.05 \uparrow$ & $0.29 \pm 0.06 \S$ \\
\hline \multicolumn{3}{|c|}{$\operatorname{Rpv}\left(\right.$ kdyne $\left.\cdot s \cdot \mathrm{cm}^{-5}\right)$} \\
\hline Baseline & $2.08 \pm 0.49$ & $2.05 \pm 0.50$ \\
\hline Maximum & $4.18 \pm 1.37 \dagger$ & $6.16 \pm 2.34 \uparrow \|$ \\
\hline \multicolumn{3}{|c|}{ Rsv (kdyne $\left.\cdot \mathrm{s} \cdot \mathrm{cm}^{-5}\right)$} \\
\hline Baseline & $13.97 \pm 2.36$ & $13.72 \pm 2.30$ \\
\hline Maximum & $15.37 \pm 3.29 \dagger$ & $16.40 \pm 3.28 \dagger$ \\
\hline
\end{tabular}

* Mean $\pm \mathrm{SD}, n=7$

$\dagger p<0.01$ vs baseline (paired $t$ test).

$\ddagger p<0.01 v$ s infant's strain (paired $t$ test).

$\S p<0.05 v s$ baseline (paired $t$ test)

$\| p<0.05$ vs infant's strain (paired $t$ test). 
Table 2. Hemodynamic responses to bolus injections of infant's strain and its mutants*

\begin{tabular}{|c|c|c|c|c|}
\hline & Infant's strain & Mutant 49-2 & Mutant 36-1 & Mutant 37-5 \\
\hline \multicolumn{5}{|c|}{$\overline{\mathrm{P}} \mathrm{pa}[\mathrm{kPa}(\mathrm{mm} \mathrm{Hg})]$} \\
\hline Baseline & $2.18 \pm 0.17(16 \pm 1)$ & $2.18 \pm 0.17(16 \pm 1)$ & $2.15 \pm 0.13(16 \pm 1)$ & $2.20 \pm 0.18(17 \pm 1)$ \\
\hline Maximum & $3.43 \pm 0.62(26 \pm 5) \dagger$ & $3.27 \pm 0.58(25 \pm 4) \dagger$ & $4.03 \pm 0.74(30 \pm 6) \dagger \ddagger \S$ & $4.07 \pm 0.72(31 \pm 5) \dagger \ddagger \S$ \\
\hline \multicolumn{5}{|c|}{$\overline{\mathrm{P}}_{\mathrm{sa}}[\mathrm{kPa}(\mathrm{mm} \mathrm{Hg})]$} \\
\hline Baseline & $8.43 \pm 0.88(63 \pm 7)$ & $8.50 \pm 1.39(64 \pm 10)$ & $8.18 \pm 0.77(61 \pm 6)$ & $8.43 \pm 1.32(63 \pm 10)$ \\
\hline Maximum & $8.47 \pm 0.83(64 \pm 6)$ & $8.52 \pm 1.28(64 \pm 10)$ & $8.48 \pm 1.08(64 \pm 8)$ & $8.33 \pm 1.04(63 \pm 8)$ \\
\hline \multicolumn{5}{|c|}{$\overline{\mathrm{Pla}}[\mathrm{kPa}(\mathrm{mm} \mathrm{Hg})]$} \\
\hline Baseline & $0.98 \pm 0.28(7.4 \pm 2.1)$ & $1.03 \pm 0.28(7.8 \pm 2.1)$ & $1.00 \pm 0.25(7.5 \pm 1.9)$ & $1.02 \pm 0.28(7.6 \pm 2.1)$ \\
\hline Maximum & $0.98 \pm 0.32(7.4 \pm 2.4)$ & $1.03 \pm 0.32(7.7 \pm 2.4)$ & $0.91 \pm 0.29(6.8 \pm 2.2) \ddagger \S \|$ & $0.94 \pm 0.35(7.1 \pm 2.6) 89$ \\
\hline \multicolumn{5}{|c|}{ Q̇pa $\left(\mathrm{L} \cdot \mathrm{min}^{-1}\right)$} \\
\hline Baseline & $0.47 \pm 0.18$ & $0.47 \pm 0.17$ & $0.47 \pm 0.15$ & $0.47 \pm 0.14$ \\
\hline Maximum & $0.44 \pm 0.16$ & $0.44 \pm 0.15$ & $0.38 \pm 0.13 \uparrow * * *$ & $0.38 \pm 0.12 \dagger \uparrow * *$ \\
\hline \multicolumn{5}{|c|}{$\operatorname{Rpv}\left(k d y n e \cdot s \cdot \mathrm{cm}^{-5}\right)$} \\
\hline Baseline & $1.78 \pm 0.94$ & $1.65 \pm 0.92$ & $1.65 \pm 0.80$ & $1.63 \pm 0.69$ \\
\hline Maximum & $3.64 \pm 1.33 \dagger$ & $3.31 \pm 1.46 \dagger$ & $5.49 \pm 2.29+ \pm \S$ & $5.53 \pm 2.69 \dagger \pm \S$ \\
\hline \multicolumn{5}{|c|}{ Rsv (kdyne. $\cdot \mathrm{s} \cdot \mathrm{cm}^{-5}$ ) } \\
\hline Baseline & $12.27 \pm 4.47$ & $12.09 \pm 5.00$ & $11.51 \pm 4.46$ & $11.47 \pm 4.43$ \\
\hline Maximum & $13.01 \pm 4.71 \dagger$ & $12.83 \pm 4.82 \|$ & $14.90 \pm 5.76+\S \pi$ & $14.64 \pm 6.17 \dagger \uparrow * *$ \\
\hline
\end{tabular}

* Mean $\pm \mathrm{SD}, n=8$.

$\dagger p<0.01 v s$ baseline value by $t$ test.

$\ddagger p<0.01 v$ s infant's strain by three-way analysis of variance with Duncan's multiple-range test.

$\S p<0.01$ vs mutant $49-2$ by three-way analysis of variance with Duncan's multiple-range test.

$\| p<0.05 v s$ baseline value by $t$ test.

$\pi p<0.05$ vs infant's strain by three-way analysis of variance with Duncan's multiple-range test.

** $p<0.05$ vs mutant $49-2$ by three-way analysis of variance with Duncan's multiple-range test.

mutant. Finally, we found a difference in effect, apparently based on capsule size, using the infant's and mother's strains that did not contain the transposon.

We showed previously that injection of GBS type III-specific polysaccharide causes little or no $\mathrm{PH}$ response in chronically instrumented piglets that have not been immunized with GBS type III (20). In contrast, injections of type III capsular polysaccharide lead to very large increases in Ppa and Rpv after $10 \mathrm{~d}$ of daily injection with heat-killed GBS type III. Type-specific antibody levels rose in conjunction with this increase in pulmonary response. This result corroborates the present conclusion that GBS capsular polysaccharide is not essential for acute hemodynamic responses in piglets and further suggests that active (and perhaps passive) immunity may alter the host's cardiovascular response to GBS bacteremia.

Gibson et al. (21) reported that a GBS type III and an isogenic nonencapsulated mutant caused similar increases in Ppa after 60 min of continuous infusion into acutely instrumented, anesthetized piglets. Their conclusion, that capsular type-specific polysaccharide is not required in GBS-induced PH, is in accord with our findings, but their results differed in that presence or absence of type-specific polysaccharide did not influence the magnitude of PH. This difference could be explained by one or more of the following. First, differing features of GBS types and strains could explain the varying experimental results. Second, the GBS strain used by Gibson et al. was reported to possess a relatively small capsule to begin with (22). Our results suggest that small capsule organisms, such as the mother's strain in the present study, cause hemodynamic effects quite similar to those caused by mutants devoid of capsule. The results of our study, therefore, suggest that one would not expect to see different hemodynamic responses when comparing a small capsule and a capsule-deficient pair. It should be noted, however, that Gibson et al. also stated that a GBS type III expressing about 10-fold more type-specific polysaccharide than their parent strain used for mutation caused increases in Ppa and $\mathrm{Rpv}$ similar to those induced by their smaller capsule GBS. Third, there may be time-related changes in PH induced by GBS (19). The data presented by Gibson et al. were at $60 \mathrm{~min}$ after beginning a continuous GBS infusion. The partially protective effect of a large capsule seen in our bolus infusion studies may disappear with time. Because no data were presented by Gibson et al. before $60 \mathrm{~min}$, one cannot draw conclusions regarding possible earlier differences between groups. Continuous infusion studies using our present GBS strains and mutants will soon resolve this issue of time-dependence for the GBS-PH response. Finally, the antibody status of the animals used by Gibson et al. may have differed from that of the animals used in our work. We have found that preincubation of GBS with type-specific rabbit antibody augments the $\mathrm{PH}$ when compared with a similar dose of untreated organisms $(18,23)$.

These studies were conducted in anesthetized, acutely instrumented, open-chested animals that were mechanically ventilated with $100 \% \mathrm{O}_{2}$. Anesthesia can blunt the pulmonary response to stimuli, including hypoxia. Despite this possible blunting, we were able to demonstrate significant $\mathrm{PH}$ effects with each organism and found clear differences between large-capsule GBS and those with small or absent capsules. Use of $100 \% \mathrm{O}_{2}$ throughout the study eliminated the possible confounding effect of hypoxia on the PH response. The animals studied by Gibson et al. (21) were ventilated with room air and developed mild hypoxemia, which may have affected the PH induced by GBS infusion. This possible effect of hypoxia may have confounded the results of their study and could even have prevented them from finding a significant difference between organisms.

It should also be noted that our studies involved the use of small, rapid injections of heat-killed GBS to assess acute hemodynamic effects of circulating organisms. Most other investigators use continuous infusions of live organisms and observe somewhat larger hemodynamic effects than those reported here. Responses to the organisms used in this study are quite similar to those previously noted by us in conscious, chronically instrumented animals (18). In our hands, continuous infusions of heatkilled organisms do lead to larger changes after 15-30 min of infusion (24). We, therefore, think that the responses noted here are consistent with those found in previous reports from our laboratory and others.

Bacterial capsules often have a protective function and are considered important in determining the pathogenicity of an organism (25). Certain encapsulated bacteria are resistant to ingestion by phagocytes and thus to destruction by the host. The lung is known to be a major site of bacterial clearance and killing. Our data suggest that luxuriant expression of capsular polysac- 
charide may further protect GBS from the host response by attenuating the pulmonary vasoconstrictive effects of incipient pneumonia and/or GBS bacteremia. Most cases of early onset GBS sepsis likely result from the aspiration of organisms from infected amniotic fluid or from passage of bacteria through mucous membranes. In either case, bacteria must traverse the pulmonary circulation before entering the systemic circulation. The spread of early invasive disease within and from the lungs might be hastened if trapping of the organisms within the pulmonary circulation was reduced. Our data suggest that heavily encapsulated organisms might induce less local pulmonary vasoconstriction than strains with smaller capsules. Such heavily encapsulated strains could then evade both immunologic and cardiovascular defense mechanisms for a longer period of time, thereby permitting a larger increase in numbers before detection by host defense mechanisms.

In summary, our results indicate that the major component(s) that causes the early hemodynamic response to GBS bacteremia is not capsular type-specific polysaccharide. Rather, an abundance of capsular material appears to partially cloak the hemodynamically active component(s) of GBS and thus inhibits, in part, the early hemodynamic responses induced by injection of GBS type Ib into piglets. Inhibition of the PH response to GBS bacteremia may increase the pathogenicity of heavily encapsulated organisms by allowing them to more readily evade pulmonary clearance mechanisms and enter the systemic circulation where they may establish foci of infection. In contrast, when less heavily encapsulated organisms do cause systemic disease in infants, they may be more likely to evoke the persistent $\mathrm{PH}$ of the newborn syndrome. This life-threatening syndrome is seen in a minority of infants with early onset GBS sepsis. The reason why this syndrome is seen in some infants with GBS sepsis but not in others remains obscure. Further studies will be necessary to determine the active component(s) that plays a critical role in triggering the hemodynamic responses observed in GBS sepsis and the importance of capsule size in the pathogenesis and manifestations of GBS sepsis in the neonate.

\section{REFERENCES}

1. Mayon-White RT 1985 The incidence of GBS disease in neonates in different countries. Antibiot Chemother 35:17-27

2. Shankaran S, Farooki ZQ, Desai R 1982 - -Hemolytic streptococcal infection appearing as persistent fetal circulation. Am J Dis Child 136:725-727

3. Walsh JA, Hutchins S 1989 Group B streptococcal disease: its importance in the developing world and prospect for prevention with vaccines. Pediatr Infect Dis J 8:271-276

4. Hageman JR, Adams MA, Gardner TH 1984 Persistent pulmonary hypertension of the newborn. Trends in incidence, diagnosis, and management. Am J Dis Child 138:592-595

5. Meadow WL, Meus PJ 1984 Hemodynamic consequences of tolazoline in neonatal group B streptococcal bacteremia: an animal model. Pediatr Res 18:960--965

6. Huddleston KW, Lyrene RK, Dew A, Gray BM, Philips III JB 1986 Influence of prostaglandin $\mathrm{D}_{2}$ on hemodynamic effects of group $\mathrm{B}$ streptococcus in neonatal lambs. Dev Pharmacol Ther 9:260-265

7. Dillon HC, Gray BM 1986 Infections caused by group B streptococci: perinatal, neonatal and early infant. In: Kelley VC (ed) Practice of Pediatrics. Harper \& Row, Philadelphia, pp 1-12

8. Ancona RJ, Ferrieri P, Williams PP 1980 Maternal factors that enhance the acquisition of group B streptococci by newborn infants. J Med Microbiol 13:273-280

9. Anthony BF, Okada DM, Hobel CJ 1979 Epidemiology of the group B streptococcus: maternal and nosocomial sources for infant acquisitions. J Pediatr 95:431-436

10. Galask RP 1988 Vaginal colonization by bacteria and yeast. Am J Obstet Gynecol 158:993-995

11. Gray BM, Pritchard DG 1991 Phase variation in the pathogenesis of group B streptococcal infections. In: Proceedings of the XIth Lancefield International Symposium on Streptococci and Streptococcal Diseases. Gustau Fischer Verlag, New York (in press)

12. Durham DL, Mattingly SJ, Doran TI, Miligan TW, Straus DC 1981 Correlation between the production of extracellular substances by the type III group $B$ streptococcal strains and virulence in a mouse model. Infect Immun 34:448-454

13. Rubens CE, Heggen LM 1988 Tn9l6 $\mathrm{E}$ : a Tn916 transposon derivative expressing erythromycin resistance. Plasmid 20:137-142

14. Macrina FL, Evans RP, Tobian JA, Hartley DL, Clewell DB, Jones KR 1983 Novel shuttle plasmid vehicles for Escherichia-Streptococcus transgeneric cloning. Gene 25:145-150

15. Clewell DB, Gawron-Burke C 1986 Conjugative transposons and the dissemination of antibiotic resistance in streptococci. Ann Rev Microbiol 40:635659

16. Håkansson $S$, Hoim S 1986 Influence of polysaccharide capsule and ionic strength on buoyant density of group B streptococci. Acta Pathol Microbiol Immunol Scand [B] 94:139-143

17. Van De Rijn I, Kessler RE 1980 Growth characteristics of group A streptococci in a new chemically defined medium. Infect Immun 27:444 448

18. Philips III JB, Lyrene RK, Godoy G, Graybar G, Barefield E, Sams JEP, Gray $\mathrm{BM} 1988$ Hemodynamic responses of chronically instrumented piglets to bolus injections of group B streptococci. Pediatr Res 23:81-85

19. Li J-X, Gray BM, Pritchard DG, Oliver JR, Foster JM, Parker CR, Philips JB 1990 Time-dependent changes in the pulmonary hypertensive response to bolus injections of group B streptococci. Clin Res 38:64A(abstr)

20. Philips JB, Huddleston C, Lyrene R, Godoy G, Pritchard DG, Graybar G, Sams J, Oliver J, Cassady G, Gray BM 1986 Role of type-specific antigen in pulmonary hypertensive response to group B streptococcus in piglets. Pediatr Res 20:401A(abstr)

21. Gibson RL, Redding GJ, Truog WE, Henderson WR, Rubens CE 1989 Isogenic group B streptococci devoid of capsular polysaccharide or $\beta$-hemolysin: pulmonary hemodynamic and gas exchange effects during bacteremia in piglets. Pediatr Res 26:241-245

22. Kuypers JM, Heggen LM, Rubens CE 1989 Molecular analysis of a region of the group B streptococcus chromosome involved in type III capsule expression. Infect Immun 57:3058-3065

23. Chwe M, Li J-X, Gray B, Pritchard D, Oliver J, Grantham K, Mena W, Philips J 1991 Effect of type-specific antiserum on the pulmonary hypertensive response to group B strococcus in piglets. Pediatr Res $29: 281 \mathrm{~A}$ (abstr)

24. Tarpey MN, Graybar GB, Lyrene RK, Godoy G, Oliver J, Gray BM, Philips III JB 1987 Thromboxane synthesis inhibition reverses group B streptococcus-induced pulmonary hypertension. Crit Care Med 15:644-647

25. Spizizen J 1984 Bacterial structure, physiology and growth. In: Sherris JC (ed) Medical Microbiology: An Introduction to Infectious Diseases. Elsevier Science Publishing Co, New York, pp 9-12 